\title{
Pre-service physics teachers' content knowledge of electric and magnetic field concepts: Conceptual facets and their balance
}

\author{
Maija Nousiainen and Ismo T Koponen \\ Department of Physics, University of Helsinki, Helsinki, Finland \\ For correspondence: maija.nousiainen@helsinki.fi
}

\begin{abstract}
The concepts of electricity and magnetism in physics are complex and demanding to learn because their meaning builds through several different phenomenological areas. Each of these phenomenological areas adds a certain facet of the meaning of the concept. All standard physics textbooks discuss at least 1) force, 2) energy and work, and 3) electric charge and current, which are different phenomenological facets of field and which also are implicitly covered in instruction. It is of interest to ask, how these three facets are actually reflected in university students' declarative (expressed and communicated) knowledge of electric and magnetic field concepts. Here this problem is addressed by using recently introduced concept networks as a research tool. Using these concept networks, pre-service physics teachers have represented their views how electric and magnetic field concepts are linked to other concepts and conceptual elements in electricity and magnetism. The results suggest that more extensive is the students' basis of content knowledge the more balanced are the facets, while students with less extensive basis of content knowledge tend to favour forcebased understanding of the electric and magnetic fields. The implications of the findings on teaching and instruction are discussed.
\end{abstract}

Keywords: field concept, conceptual facet, concept network, conceptual understanding

\section{Introduction}

The concepts of electric and magnetic field are known to be difficult to learn. Possible reasons for the widely recognized learning difficulties have been approached from many vantage points: Electric field related to force and action-at-a-distance (Furió \& Guisasola, 1998; Galili, 1995), relationship of electric field and electric charge as its source (Guisasola et al., 2008; Viennot \& Rainson, 1992), and different representations of field, like field lines (Törnqvist et al., 1993; Pocovi \& Finley 2002). Similar kind of problems in learning about magnetic fields have also been reported (Guisasola et al.,2004; Albe et al., 2001). In each of these studies some specific aspect of field concept and some specific problems in learning of those facets, is illustrated and discussed. To these specific, separate but essentially connected aspects we refer as "facets". In addition to studies focusing on certain specific facet of field-concepts also the students' understanding of the relationships between the different facets have been explored (Savelsbergh et al., 2011). In summary, the previous studies strongly suggest that it is useful to think the field concept as a complex and many faceted concept (Albe et al., 2001) and, therefore, also approach the learning of the field concepts from an integrated viewpoint where different facets are taken into account simultaneously.

The relevant facets we are focusing on here are force, energy and source (charge or current). These facets are discussed in standard textbooks (see e.g. Knight, 2008; Halliday et al., 2005; Young \& Freedman,2004) as well as in textbooks which have attempted to reform the traditional approaches(see e.g. Chabay \& Sherwood, 2002; Feynman et al., 1964). In introductory level, teaching of electric and magnetic field starts often with force based phenomenology, discussing the forces (and 
torques in case of magnetic field) caused by electric or magnetic interaction (see e.g. Knight, 2008; Halliday et al.,2005). This is called here the force-facet. Then, the idea of source creating the field, electric charge for electric field, and electric current for magnetic field, is introduced. This can be referred as source-facet. The third facet, the energy-facet, is related to work and energy, usually introduced through potential energy of electric or magnetic interaction. In case of magnetic field the preferred starting point is often the electric current as source of the field (see e.g. Knight, 2008; Chabay \& Sherwood, 2002), and to a lesser extent, force- and source based facets are introduced. In textbooks, these three facets, however, are not explicitly addressed as different paths and alternative but complementary ways to build the meaning of field concept.

The three-faceted nature on electric and magnetic fields as it appears on the textbooks have its roots in history of physics (for historical details see e.g. Whittaker, 1910; Darrigol, 2000). Even today, these historical roots can be easily discerned in textbooks. However, the questions of historical roots and their relation to textbook presentations are not pursued here further. It is sufficient to note that thinking the field concept as multifaceted is well motivated both from the viewpoint of current teaching and instruction, as well as point of view of history of physics.

The question addressed here is if the facetedness is present in students' representations of their knowledge about the electric and magnetic fields and if it is balanced or focused only on certain facets. Previous research namely suggests that very often students' conceptions of field concepts are forced-centred (Furió\& Guisasola, 1998).

Consequently, the research question posed here is

RQ: How the facets of force, energy and source appear in pre-service teachers' declarative knowledge of the electric and magnetic field concepts?

In order to provide answer to this question, we need to have: 1) representational tools that allow students to represent the multifaceted aspects of their knowledge, and 2) appropriate methods of analysis to discern the multifacetedness. Here, the recently introduced concept networks and the guidelines to construct them (Nousiainen, 2013) are adopted as representational tools. Such networks are shown to allow students to visualize complex relational aspects of their knowledge, but also requiring careful justification of the knowledge which is represented (Nousiainen, 2013; Koponen \& Nousiainen, 2013).

The sample of the study consists of concept networks made by 12 university students (third year preservice physics teachers). The analysis then builds on networks analysis, paying attention on the global connectivity of the network as recently suggested (Koponen \& Nousiainen, 2014). The results of the study show that richness of the content of the students' knowledge of the different facets of the electric and magnetic field and the balance between the different facets are correlated: more students know other concepts, models and experiments related to the field concept, the better are the different facets balanced. Also, there is clear difference between electric and magnetic field concepts, both in the richness of content and how different facets are weighted.

We show that the approach paying attention on facets is able to reveal important differences in students' declarative knowledge, reflecting possibly different phenomenological profiles of their understanding. As far as conceptual understanding is concerned, such information alone is valuable in gauging and monitoring students' conceptual knowledge. Whether or not the differences in balance of facets are reflected in e.g. problem solving abilities is left as an open question for future research. 


\section{Facets of electric and magnetic fields}

Inspection of physics textbooks for first year university students reveals that electric field is taught so that it is first approached mainly from viewpoint of action-at-a-distance force, typically introducing the static electric force and Coulomb's law (see e.g. Knight, 2008; Halliday et al.,2005; Young \& Freedman,2004; Majidi, 2014; Majidi \& Mäntylä, 2011). Many suggested laboratory and lecture demonstrations to teach static electricity also actually illustrate and support the action-at-a-distance interpretation (Chang, 2001). The action-at-a-distance viewpoint emphasize the Newtonian view on mutual interactions, where field appears as a description of the strength of the interaction attributed to the only one agent (object), usually to the more powerful or influential one and defined through relation E=F/q in case of electric field. However, conceptually such a definition of field alone adds little to the force concept and such field concept remains in many respects redundant to force (cf. Furió \& Guisasola, 1998).

After the preliminary introduction of field through force the second step taken in the textbooks is often to introduce electric potential energy and electric potential, often through application of mechanical work and conservation of energy (see e.g. Knight, 2008; Halliday et al., 2005; Young \& Freedman, 2004). This path of instruction is targeted to the introduction of electric potential energy and electric potential and how they are related to the field. Historically, this approach to augment the meaning content of electric field matured in middle of 19th century simultaneously with maturation idea of the centrality of conservation of mechanical energy (Darrigol, 2000; Whittaker, 1910). Textbooks, however, do not underline the different conceptual basis of the force based and energy based introduction of field although this distinction is clearly visible in the way the field concept is approached in different chapters of the textbooks.

The third step common to all textbooks is the introduction of field as originating or "emanating" from electric charge, a view also closely related to the Newtonian action-at-a-distance picture of field, but historically developed fully by Gauss in early 19th century (Darrigol,2000; Whittaker, 1910). In this context the textbooks usually introduce the Gauss' law that describes the relation of charge and field. In this context at least implicitly field is often treated as an independent, space filling entity created by charged body. These three different phenomenological viewpoints, although closely related, can be thought as force-, energy and source-type phenomenological basis, respectively, to describe and understand different facet of concept electric field, and they are thus referred here as force-, energyand source-facets.

The textbook approaches to magnetic field differ in certain respect from the approach to electric field. Moreover, between different textbooks there are more remarkable differences in their approaches in case of magnetic field while approaches to electric field tend to be more similar from one textbook to another (see also Majidi \& Mäntylä, 2011). In textbooks, the magnetic field (or magnetic flux density) is often introduced first through introductory notions of force or torque related to magnetic interaction, but then, quite in the beginning of the discussion, magnetic field is understood as created by electric current, as described by Ampère and Laplace law (misnamed in most US textbooks as BiotSavart law, for correct terminology see e.g. Whittaker, 1910), and later on, through Ampère's circuital law (Knight, 2008; Young \& Freedman,2004). This stands for sourcebased phenomenology, introducing to student the sourcebased facet of magnetic field. The force or torque based facet is featured in all textbooks to some extent, very often first by using the barmagnets. Consequently, the case the sourcebased introduction is then often mixed with forcebased action-at-a-distance based explanations (Knight, 2008; Young \& Freedman, 2004) while in some cases clear-cut priority is given to purely source based introduction (Chabay \& Sherwood, 2002). The energybased facet is not always very thoroughly discussed; if done, it appears most often in context where coil is turned in external 
field, leading to introduction of potential energy between coil and field. Nevertheless, also in case of magnetic field we can recognize the force, energy and source facets.

It is of interest to note that the textbooks presentations follow in many respects the historical order of the field concept was discovered. Historically the action-at-a-distance description, where force was connected to interaction of two bodies and acting through empty space and the force, related to the certain characteristic property of the bodies (in case of electric field, to charge, in case of gravitational field to mass, and in case of magnetic field to magnetic moment). The action-at-a-distance description of electrical and magnetic interactions was also the basis of Ampere's way to describe the electric and magnetic fields and on this basis, he constructed his theory of electricity and magnetism (Darrigol, 2000). An approach by Gauss to action-at-a-distance forces attributes the field to single body and at least implicitly described the field as emanating from the single body, filling the space and pervading to everywhere. This way of though was of course also present in Newton's idea of field but Gauss formalized and explicated this idea through mathematical theory. The connection of energy and work to electric field is closely related to the potential theory of fields, which culminated in Thomson's (later Lord Kelvin) formulation the theory of electricity when he set the foundations of potential theory of electric and magnetic fields and established the connection of electric potential and electric potential energy to energy conservation principle (Darrigol, 2000; Smith \& Wise,1989). Therefore, the force, energy and source facets can be also discerned in historical development of electric and magnetic field concepts.

Without discussing more about the way the textbooks present the electric and magnetic field concepts (for more details, see Majidi, 2014; Majidi \& Mäntylä, 2011) or the historical origins (for comprehensive accounts, see Whittaker, 1910; Darrigol, 2000) we refer to the force, energy and source facets of electric basis as symbols $\mathrm{EF}_{\mathrm{F}} \mathrm{Ew}$ and $\mathrm{Es}$, or briefly as E-field facets, and similarly for magnetic field (or more correctly magnetic flux density, but this distinction is not made here) as $\mathrm{BF}$, $\mathrm{Bw}$ and $\mathrm{Bs}$, briefly as B-field facets. The basic assumption here is that complete understanding of static electric and magnetic fields requires understanding all these three facets. In particular, the force-centred approach in field concepts is doubtful if extended too far and if presenting too long.As a sole basis of students' understanding such an approach on fields have little basis in modern picture of physics. For example, in QM electromagnetic field enter through theory energy facets, as potentialfunctions and in form potential theory. In most applications, on the other hand, the approach based on vector analysis (i.e. field theory essentially in form Thomson introduced it, utilizing the preliminary formulation of Gauss) is central. Apparently for these reasons some textbooks (e.g. Chabay \& Sherwood, 2002) have attempted approaches based rather on the sourcethan on the force facet. Therefore, the hypothesis motivating and guiding this study is that these different facets of electric and magnetic fields may be balanced very differently in case of different students and that not all students have not necessarily gained a balanced view, where all three facets are equally featured.

\section{Empirical sample: Concept networks}

The empirical sample of the research reported here is collected from an intermediate-level course for pre-service physics teachers (third or fourth year students). During the course, the students reflected of their knowledge of electricity and magnetism from the point of view of teaching these subjects in upper secondary school. All students had completed a basic course in electricity and magnetism, in level discussed e.g. in a standard textbook (e.g. Knight, 2008). However, many of the students had completed the basic EM course with rather poor or moderate grades and in the beginning of the teacher preparation course the CSEM test revealed rather moderate pre-test scores; 5 out 12 under $50 \%$ of maximum scores and only 4 better than $80 \%$ of maximum scores. 
The sample of this study consists of 12 concept networks with written reports. The networks and written reports are made by students representing their ideas of how concepts of electricity and magnetism are interlinked. The students constructed the concept networks individually during a seven week course and the working was organized in three stages. In the first stage, an initial version of concept network and report was produced. The second stage was improving the networks by group discussions and peer-feedback. Some formal lectures about the topic was also given by instructor. The last stage was the final version of the network. The networks were made about three different topics being 1) electric field, 2) magnetic field and 3) induction. During the seven week course students had about two weeks' time to start, improve and finalize each topic. The third network (about induction) link together electric and magnetic field concepts. Therefore, the final third networks contain all the concepts, conceptual elements and their relation students eventually found relevant. Here, only these extensive networks including all field concepts are considered.

Design principles of concept networks rest on the use of quantitative experiments and models which are the traditional and important parts of science teaching (Koponen \& Nousiainen, 2013; Nousiainen, 2013). In the quantitative experiment a concept is operationalized, that is, made measurable through pre-existing concepts. For example, the operationalization of Coulombs' law requires application of the concepts of force and charge, whereas the concept of electric flux rests on electric field, charge distribution and field line model, and so on. In such experiments, a new concept or law is always constructed sequentially, starting from ones that already exist, and which therefore also provide the basis for experiment design and interpretation. In addition to experiments, models are also core components of knowledge structures. For example, the definition of electrical field can be seen as a model which interprets the force between two interacting charges as an interaction between two parts; one which causes the field (the charge as a source) and another which experiences the field (the other charge). Another example is the model of a unified field, extensively used as a model in introductory electricity courses. Typically, a model may be an idealized and symbolic representation or a description of dependencies found in an experiment or that should provide explanations and predictions of regularities found in experimental data.

Consequently, the design of the concept networks discussed here is based on special kinds of nodes representing the knowledge. The nodes in these networks represent:

1. Quantities

2. Laws

3. Models

4. Experiments

5. General principles

Of these elements, laws are either particular experimental laws or law-like predictions in specific situations (derived from a theory) or general laws which are more fundamental principles (e.g. principles of conservation). A general principle is e.g. energy conservation principle or superposition principle for e.g. fields. The relations between nodes are established through different types of procedures or actions: in the case of an experiment, for example, the quantity can be changed, measured or kept constant; in the case of a model, the model can predict or constrain a given quantity. The linking words are expected to describe these possible procedures and actions. The basic idea is that the design principles guide the construction process of the network. It should be noted that students must describe and give epistemic justifications for each node-link-node chain they draw and write them down in the attached report explaining the concept network.

The design method reported here was developed for the purposes of pre-service physics teachers' training courses, and with gradual improvements it has been in use on such courses since 2008. In practice, it has given students a framework to develop their conceptual understanding. Results 
related to the practical use of the networks are discussed in more detail elsewhere (Koponen \& Nousiainen, 2013; Nousiainen, 2013). However, two important modifications were made in the design rules as they are explained in Koponen and Nousiainen (2013) and Nousiainen (2013). First, the models and experiments that students use to introduce or support the concepts, are now represented as nodes. The links contain now only a description of the type of action or procedure (definition, measurement, quantification etc.) how nodes are connected. For example, when model-node is used to support introduction of concept-node, a definition is often used. Second, students were asked to pay special attention on the field concepts. Students were asked to display in the network separately what they thought to represent the force, energy and source supported parts of electric and magnetic fields (i.e. different facets) but with the understanding that complete field concept contains all these three facets (also fourth facet, dynamic, is included in the induction network but dynamic facet is not discussed here). This results on representations where each facet is presented separately and same connections can appear in case of more than one facet. It is important to note that this is only a representational choice, made for clarity of reading of the resulting networks, and does not bias or restrict the way the students are allowed to construct the connections in the concept networks.

An example of concept network produced by a student, following the design principles described above, is shown in Figure 1. In the network the nodes representing the different facets of field concepts are shown as bolded boxes. Some of the most important concepts, laws, experiments and models, and how they are classified as force, energy or sourcebased are given in Table 1.

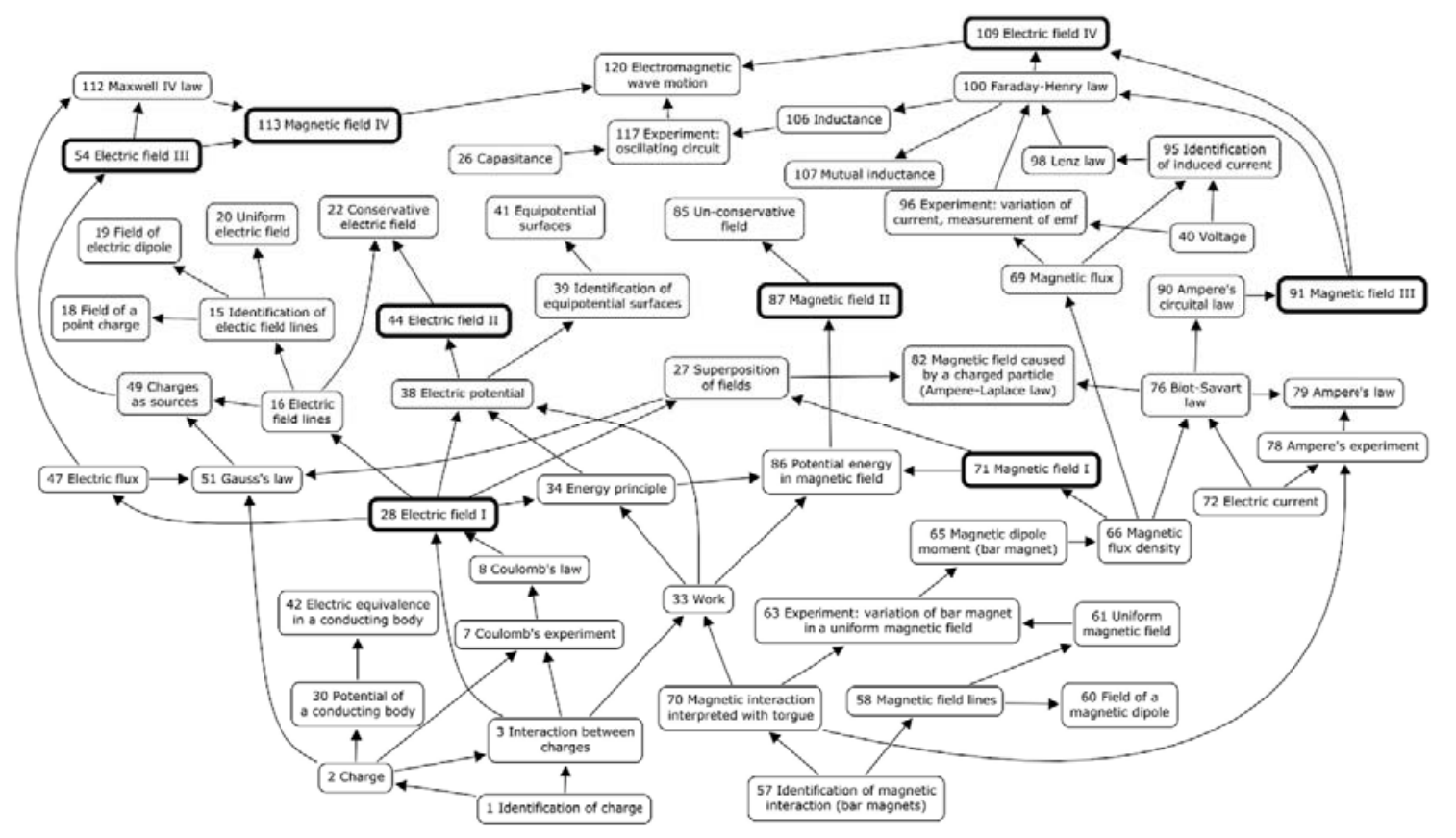

Figure 1.An example of the concept network of 55 nodes made by one pre-service student (redrawn and translated for clarity). The nodes with bolded borders are different facets (force, energy and sourcefacets, I-III respectively) of field concept. Note that fourth facet (dynamic, IV) is also shown but not considered further here.

Each of the 12 concept network is different in regard to number of nodes, which varies from 60 to 90 , and in regard to links, which varies from 70 to 110. Taken together, in all 12 maps there are 121 different nodes and about 400 different links. Different concept networks may have very different collection of nodes. However, the three E-field facets and three B-field facets appear in all 12 networks. 
Table 1. Examples of concepts and other conceptual elements appearing in concept networks and as they are classified as force, energy and sourcebased classes. Only some most central concepts and conceptual elements out of all 121 ones found in the 12 networks are listed.

\begin{tabular}{lll} 
Force facet & Energy facet & Source facet \\
\hline $\begin{array}{l}\text { Force } \\
\text { Coulomb's law }\end{array}$ & Work & Electric charge $Q$ \\
Coulomb's experiment & Work done to move a charge & Millikan's experiment \\
Gravitation law (analogy) & Energy conservation & Charge distribution \\
Field-line experiment & Potential energy $V$ & Gauss's law \\
Electric dipole (force on) & Electric potential $U$ & Electric flux density $D$ \\
Point charge (force on) & $E=-$ grad $U$ & Electric flux \\
Homogeneous field & Equipotential surface (exp.) & Electric current $I$ \\
Electric force $F=q E$ & Voltage & Electric current density \\
Electrostatic equilibrium & Work done to rotate a coil & Magnetic dipole moment \\
Torque & Magnetic potential energy & Ampere and Laplace law \\
Force/torque between magnets & electromotive force (e.m.f.) & Biot-Savart law \\
Ampere's law & Power related to induction & Biot-Savart experiment \\
Ampere's experiment & B as non-conservative field & Ampere's circuital law \\
Lorentz's force & Resonance circuit (exp) & Ampere's circ. law (model) \\
& Energy in resonance circuit & Current element
\end{tabular}

\section{Method of analysis}

The analysis is a combination of qualitative and quantitative methods. This section summarize first the qualitative method of epistemic analysis, which follows guidelines given by Nousiainen (2103) and then the quantitative analysis which is based on network theory as developed further and explained in detail by Koponen and Nousiainen (2014). Details of these methods are summarized here only to extent that is needed to understand how these methods of analysis can be then used to identify the different facets of field concepts.

Qualitative Analysis: Epistemic Justification. The question of the epistemic validity of knowledge concerns the epistemic acceptability of explanations students provide in their written reports. In order to analyse epistemic justification a suitable taxonomy is needed. There are several options for this, differing in the ways they emphasize domain specific content in relation to general content (Kelly \& Takao, 2002; Krathwohl, 2002; Sampson \& Clark, 2008; Sandoval \& Millwood, 2005). In the present study attention is paid only to following four epistemic aspects of knowledge: 1) ontology, 2) facts, 3) methodology and 4) valid justification (Nousiainen, 2013). The criteria are such that they are nearly self-evident prerequisites of acceptable knowledge in physics. Therefore, these four criteria form a suitable basis for the analysis of epistemic acceptability of knowledge represented in the written reports coming with concept networks:

1. Ontology. Concepts are applied properly and they describe ontologically correct entities. Ontology also requires that the properties a quantity has are described adequately and that concepts are correctly related.

2. Facts. Appropriate and correct concepts are connected by presenting appropriate and correct quantities and laws that establish the connection. For a law or principle, the proper relation is given in a clear manner.

3. Methodology. In this approach methodological knowledge is limited to knowledge including the description of such experiments and models, which are feasible to make connections between physics quantities and laws. 
4. Valid justification. Justified knowledge means skills to give ontologically correct, factually acceptable, and methodologically plausible arguments. For an experiment, this means that the prerequisites for the experiment are clearly and correctly described, that the experiment itself is adequately described (what is measured and how), and that results are expressed and conclusions discussed at some length. A similar sequence can also be followed in evaluating a model.

These criteria form cumulative, hierarchical ladders and thus the numbering (1-4) is indicative of the order in which the above epistemic "norms" should be fulfilled.

In addition to the epistemic analysis of the nodes, also the links were evaluated using the following taxonomy:

1. Only a drawn line or a meaningless linking word which does not present any new information (e.g. "is" or "connection").

2. Ambiguous linking word which does not fit in between the nodes (e.g. "illustrates", "utilizes"). Proposition is somewhat right but scientifically poorly expressed.

3. Descriptive and suitable linking word which connects logical concepts (e.g. "defined", "measured", "varied", "kept constant"). Proposition is right and scientifically sound (cf. Yin et al., 2005).

Note that the epistemic analysis of written reports and linking words is the only interpretative part of the analysis. It produces the data for the quantitative analysis in the form of epistemic weights of nodes and links in the concept networks.

In order to analyse how different facets of field concepts emerge from the relationships between nodes in the network, we classify each node on basis how it is related to force (or torque), energy (or work), or source (electric charge or current). Of course, many nodes have one or more of these characteristics, but still it is relatively straightforward to recognize the dominant phenomenology. For example, to forcebased class (F) belong: Coulomb's law for a force between charged bodies, Ampère's law for two wires carrying a current, and Lorentz's force. To energybased class (W) belong: Model describing work done when charged body is moved in homogeneous electric field, work done when coil is turned in homogeneous magnetic field, and definitions of corresponding potential energies of interaction. In sourcebased class(S) one finds: Gauss's law, Ampère's circuital law, Ampère's and Laplace's law. In this way, most of the nodes can be "tagged" to belong one of three phenomenological classes: Force or torque (F), Energy and work (W), Source, which is either electric charge or current (S). The nodes in classes $\mathrm{F}, \mathrm{W}$ and $\mathrm{S}$ thus form three tagged modules of nodes. However, about $15 \%$ of nodes cannot be classified in any of these modules.

In the network, the field concepts $\mathrm{E}$ and $\mathrm{B}$ are represented so that the those connection of field concepts, which are thought to be most closely related to force (F), energy (W) or source (S), are represented as separate nodes denoted in what follows by eF, ew and es for electric field and as $b_{F}$, $b_{W}$ and bs for the magnetic field (or magnetic flux density). The complete phenomenological support and thus the facet strengths Ex and $\mathrm{Bx}$ (where $\mathrm{X}$ is $\mathrm{F}, \mathrm{W}$ or $\mathrm{S}$ ) of the electric and magnetic field concepts, respectively, are then formed of the F, W and S support of nodes ex and bx. This is done by counting the suitably weighted paths from these nodes to all other nodes belonging to given phenomenological module $\mathrm{F}, \mathrm{W}$, or $\mathrm{S}$ of other nodes. Depending on how the given facet is connected to a given module, we can decide how the complete phenomenological support of fields E and B are actually composed. Details and formalization of this path counting are explained later on.

The qualitative analysis and interpretations of the content of nodes and links were carried out by two interpreters. From the sample about one third of nodes and links were picked out in random and was 
analysed by second interpreter. The agreement of interpreters was better than $75 \%$, and differences were limited to \pm 1 in differences in the categorization. Such differences with level $25 \%$ disagreement is nearly insignificant in the final analysis.

Quantitative analysis. In order to find out how nodes representing different facets of field concepts are supported by force, energy and sourcetype concepts, we must find out how they are connected to nodes which are "tagged" to belong one of the three modules. In order to this, we must define and operationalize the property of "being connected". It should be noted that here local connectedness, i.e. the number of links in given node or average number of links are simply inadequate measures. Instead, global measures of connectivity, which takes into account the chains of connection or extended connecting paths, needs to be used (c.f. Koponen and Nousiainen 2014). For this, the information contained in the concept network itself must be suitably formalized so that the network yields to quantitative analysis.

After the interpretative analysis, the important information contained in the networks is carried by node and link strengths. For practical reasons all the values were normalized to range from 0 to 1 . Epistemically strong nodes and links have strengths $0.75-1$, while weak nodes and links have small values $0.0-0.25$. The strength of node $i$ is denoted by $\mathrm{si}$, while $\mathrm{w}_{\mathrm{ij}}$ is the strength of the link from node $\mathrm{i}$ to node $\mathrm{j}$. The information on epistemic strengths is simplified in further analysis by rescaling link strengths so that the epistemic strength of an initiating node and a link emerging from it are aggregated to form a new weight $\mathrm{w}_{\mathrm{ij}} \rightarrow \mathrm{si}_{\mathrm{i}}(\tau) \mathrm{W}_{\mathrm{ij}}$, as exemplified in Figure 2 (also see Figure 1).

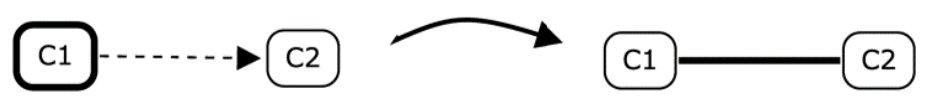

Figure 2. The reweighting of the network. Weights of preceding node $\mathrm{C} 1$ and link are combined and the aggregate of them is denoted with the weight of the link.

This rescaling is motivated also by the notion that directed links $\mathrm{W}_{\mathrm{ij}}$ pass supporting information from node $i$ to node $j$. When rescaling is done, the epistemic support and its directed nature is taken into account in the node strengths, and the links can be symmetrized $\mathrm{w}_{\mathrm{ij}}=\mathrm{w}_{\mathrm{ji}}$. After rescaling, the node strengths are set to the value of 1 , and directionality is removed. In addition to strength, the node carries a tag $\tau$ which specifies the type of the node, either force $(\tau=F)$, energy $(\tau=E)$ or source $(\tau=S)$.

The essential information of content of the concept networks is coded on the weights $w_{p q}$ of the links connecting nodes $\mathrm{p}$ and $\mathrm{q}$. The weights for $\mathrm{N}$ nodes forms a $\mathrm{NxN}$ weighted adjacency matrix $\mathrm{W}$ with elements $[\mathrm{W}]_{\mathrm{pq}}=\mathrm{W}_{\mathrm{pq}}$. Here, a weighted adjacency matrix is used in order to take into account the epistemic strengths of links.

The phenomenological support of given facet of the field concept can now be calculated on basis how it is connected to other concepts belonging to modules. It can be assumed that facet $\mathrm{F}$ is dominantly connected to F type concepts, facets W and S to Wand Stypes, respectively. However, all other crossconnections are also possible. In order to find out the connections, we need to calculate all possible weighted paths from concepts of a given type to the given facet. For example, the F, W and Sbased support of $\mathrm{EF}_{\mathrm{F}}$ is obtained by counting all paths from $\mathrm{F}, \mathrm{W}$, and Stagged nodes to node $\mathrm{EF}_{\mathrm{F}}$, and similarly for all other field concept facets. In counting the paths, we need to pay attention that the number of possible long walks tends to increases factorially, and thus simply calculating the paths would bias the result towards the possible alternative routes. Therefore, long walks are weighted by inverse of factorial in order to reveal their relative importance in connecting nodes (Koponen \& Nousiainen, 2014). 
The weighted matrix can be used directly to obtain the number of walks. This is based on notion that there is a walk from $\mathrm{p}$ to $\mathrm{q}$ if $\mathrm{w}_{\mathrm{pq}} \neq 0$, walk $\mathrm{p} \rightarrow \mathrm{j} \rightarrow \mathrm{q}$ if $\mathrm{w}_{\mathrm{p},}, \mathrm{w}_{\mathrm{jq}} \neq 0$, walk $\mathrm{p} \rightarrow \mathrm{k} \rightarrow \mathrm{k}^{\prime} \rightarrow \mathrm{q}$ if $\mathrm{w}_{\mathrm{p}}, \mathrm{w}_{\mathrm{j} k}, \mathrm{w}_{\mathrm{kq}} \neq 0$ etc. On the other hand, for walk involving two nodes $W 2 \neq 0$, for three nodes $W 3 \neq 0$, respectively. Now, in connected network, the number of long walks increases rapidly, nearly factorially with the length of the walk, because different combinatorial possibilities emerge, and one is interested of the relative weight of such walks. Therefore, the number of walks is usually divided by the factorial, to obtain.(Estrada, 2012; Koponen \& Nousiainen, 2014)

$$
G_{p q}=1+\frac{[W]_{p q}}{1 !}+\frac{\left[W^{2}\right]_{p q}}{2 !}+\frac{\left[W^{3}\right]_{p q}}{3 !}+\cdots=\left[e^{W}\right]_{p q}
$$

This counting of walks is adopted as a basis to operationalize the phenomenological support of given facet of field concept. In calculating the support $\mathrm{Y}$ (where $\mathrm{Y}$ is $\mathrm{F}, \mathrm{W}$ or $\mathrm{S}$ ) of facet $\mathrm{Ex}$ (where $\mathrm{X}$ is $\mathrm{F}, \mathrm{W}$ or S), we use $\mathrm{G}_{\mathrm{pq}}$ to obtain effective path strengths $\gamma$ for node $\mathrm{p}$ representing the given field facet with $\tau=X$ and taking a sum over all other nodes $q \neq p$ with $\tau=Y$, to obtain

$$
\gamma_{p}(X ; Y)=\log \sum_{q} G_{p q} \text { where } \in \in X \text { and } q \in Y, \quad q \neq p
$$

The effective path strength corresponds roughly the number of links equivalent to strength 1 that connect the node $\mathrm{p}$ of type $\mathrm{X}$ to type nodes of type $\mathrm{Y}$ in the network. Typically $\mathrm{G}_{\mathrm{pq}} \sim 10$ for an effective path which is of significance. A few paths has $0 \mathrm{Gpq}_{\mathrm{pq}}<1$ and these are set to minimum value 1 to allow a connection to be counted.

The quantity $\gamma$ is called the Communicability Centrality of node p. With this definition, the essential information of the support $\mathrm{Y}$ of given field facet is contained on the total communicability centrality $\Gamma \times$ of the node representing that facet $\mathrm{X}$, calculated for all nodes belonging to module $\mathrm{Y}$ as

$$
\Gamma_{X}=\sum_{Y, p \in Y} \gamma_{p}(Y ; X)
$$

The total communicability centrality $\Gamma x$ (or more briefly centrality in what follows) can be then used to quantify the F-, W- and S-facets when one identifies communicability centralities $\gamma_{\mathrm{p}}$ with nodes representing eF, ew or es in students' networks case of E-field and with $b_{F}$, $b_{w}$ and $b$ s in case of B-field. It should be noted that in the student maps ex and bx (where $\mathrm{X}$ is $\mathrm{F}, \mathrm{W}$ or $\mathrm{S}$ ) are represented, not Ex and $B x$. The nodes ex and $b x$ in maps are just denotation to keep the representation simple and transparent and avoid cluttering of nodes, while the total communicability centralities Ex and Bx calculated from networks reveal the strength of conceptual support and thus strength of facets.

The facet strengths Ex and $\mathrm{Bx}$ (where $\mathrm{X}$ is $\mathrm{F}, \mathrm{W}$ or $\mathrm{S}$ ) of electric and magnetic fields, respectively, can be associated now immediately with total communicability centralities (i.e. centralities in what follows) as $E x=\Gamma x$ when $\gamma_{p}$ is ef, ew or es and as $B x=\Gamma x$ when when $\gamma_{p}$ is $b_{F}, b w$ or bs. In addition, when each facet is interpreted as a dimension with a value given by the centrality $\Gamma x$ then the norm

$$
\Gamma=\left(\sum_{X} \Gamma_{X}^{2}\right)^{1 / 2}
$$

can be taken as a measure the richness (or total amount) of declarative knowledge the student has expressed in the concepts network. Note that these measures take into account the epistemic justification the knowledge so that large values require well many well justified connections.

The centrality of a given facet of field concept gives information how the facet is supported by different types of nodes of the whole network of nodes (concepts, laws etc.) in global scale. It takes into account all possible paths, but weights the connections so that more distant (more steps there are 
separating the nodes from each other) the node is, the smaller is the weight of that path. In comparison to local centrality measures, like e.g. connections to neighbouring concepts or connection to neighbours and nearest neighbours, such global measure is superior in picking out the key connections, as we have shown previously (Koponen \& Nousiainen, 2014). However, for networks of tens of concepts and links such analysis is not feasible by manual counting of connections and fully computational counting is needed, because the number of possible paths increases rapidly with increasing number of nodes. For example, the concept networks analysed here contain usually 60-90 nodes, with 70-121 links, and then possible paths amounts up to thousands.

\section{Results}

Students' declarative (i.e. expressible and thus communicable) knowledge of electric and magnetic field concepts as it is expresses in concept networks (see Figure 1) is here condensed in the centralities $\mathrm{EF}_{\mathrm{F}}$ Ew and Es of electric field facets and the centralities $\mathrm{BF}, \mathrm{Bw}$ and $\mathrm{Bs}$ of magnetic field facets obtained through analysis of the networks as exemplified in Figure 1. The centralities represent thus the strengths of the different facet of field concepts. Students' declarative knowledge of the three facets of two fields can be represented as a six-dimensional vector $\Gamma=(\mathrm{EF}, \mathrm{Ew}, \mathrm{Es}, \mathrm{BF}, \mathrm{Bw}, \mathrm{Bs})$. This six-dimensional information of the field concepts is conveniently and transparently expressed in form of hexagonal spider webchart, where each corner of hexagon represents one dimension. Three examples how spider webgraphs are used to represent the E- and B-field facets are shown in Figure 3.
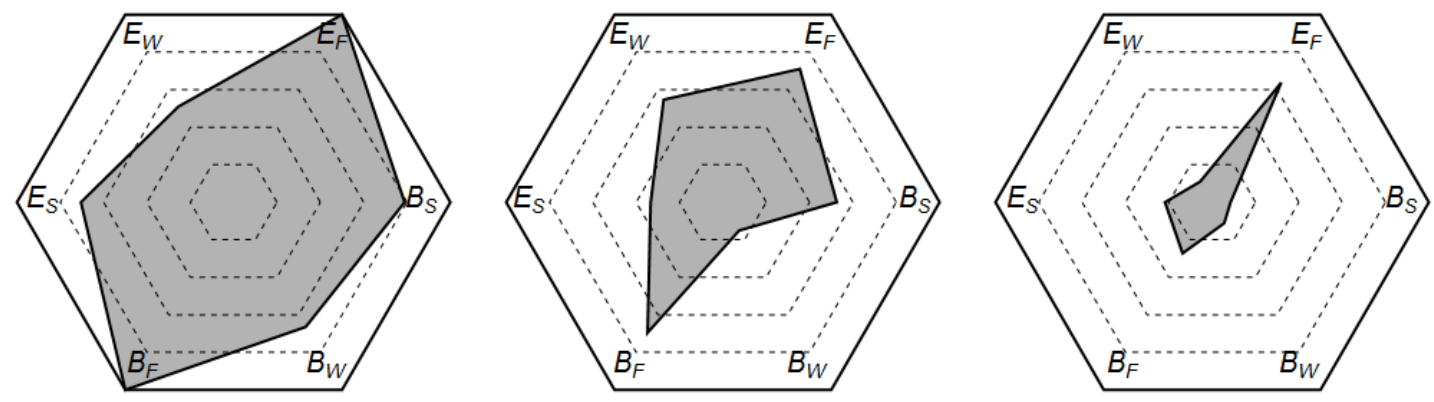

Figure 3. The six-dimensional vector $\mathrm{G}=\left(\mathrm{EF}_{\mathrm{F}}, \mathrm{Ew}, \mathrm{Es}, \mathrm{B}, \mathrm{Bw}, \mathrm{Bs}\right)$ consisting of facets $\mathrm{F}, \mathrm{W}$ and $\mathrm{S}$ of $\mathrm{E}-$ and B-fields represented as hexagonal spiderwebchart. The dashed lines locate the values $0.8,0.6,0.4$ and 0.2, while the bold line represents value 1.0. Three examples are shown: $\mathrm{E}$ and B-fields with strong facets $\mathrm{F}$ and $\mathrm{S}$ facets (left) and F-facet dominant (middle), and dominant F-facet E-field (right).

The facets of E and B fields obtained from all 12 students' concept networks are shown in Figure 4 as hexagonal spider webcharts, coded as shown in Figure 3. Because the E and B facets are conceptually and phenomenologically different, we have normalized the E and B facets separately so that a facet with a maximum value of centrality has always a value 1 for both fields separately (case at left in Figure 3, case A in Figure 4). The non-normalized values of case A corresponding the value 1 of $\mathrm{E}$ field is 8.7 and that of B field 10.2. These values correspond the effective, average number of fully justified links supporting the given facet (F-facets in case of A). All other values for E facets and B facets shown in Figure 4 are relation to these values, respectively.

Roughly, the areas of charts reflects the richness of content, i.e. larger the area, more links which are well-justified. More accurately, the richness (i.e. the amount) of declarative knowledge is given by the norm $\Gamma$ of centralities in Eq. (4). For E- and B-field the norm $\Gamma$ is denoted as $\Gamma_{\mathrm{E}}$ and $\Gamma_{\mathrm{B}}$, respectively. The richness of content as calculated from the norm is provided in Table 2 (later seen also in Figure 5) for E and B facets separately, and for combined E and B (norm of the total six-dimensional centrality vector) for all 12 cases is also reported 
Table 2. Richness of content as norm $\Gamma$ of centralities for all 12 cases A-L. For E- and B-field the norm $\Gamma$ is denoted as $\Gamma_{\mathrm{E}}$ and $\Gamma_{\mathrm{B}}$, respectively. The total richness for all facets is given by $\Gamma_{\mathrm{E}+\mathrm{B}}$.

\begin{tabular}{lllllllllllll}
\hline & $\mathrm{A}$ & $\mathrm{B}$ & $\mathrm{C}$ & $\mathrm{D}$ & $\mathrm{E}$ & $\mathrm{F}$ & $\mathrm{G}$ & $\mathrm{H}$ & $\mathrm{I}$ & $\mathrm{J}$ & $\mathrm{K}$ & $\mathrm{L}$ \\
\hline$\Gamma_{\mathrm{E}}$ & 11.5 & 7.9 & 8.3 & 7.5 & 8.6 & 6.1 & 5.9 & 5.9 & 4.8 & 4.3 & 3.2 & 2.0 \\
$\Gamma_{\mathrm{B}}$ & 14.6 & 14.9 & 9.0 & 4.6 & 5.5 & 5.6 & 3.3 & 3.1 & 4.3 & 4.6 & 1.9 & 1.2 \\
$\Gamma_{\mathrm{E}+\mathrm{B}}$ & 18.6 & 16.9 & 12.2 & 8.8 & 10.2 & 8.3 & 6.7 & 6.7 & 6.5 & 6.3 & 3.7 & 2.4 \\
\hline
\end{tabular}
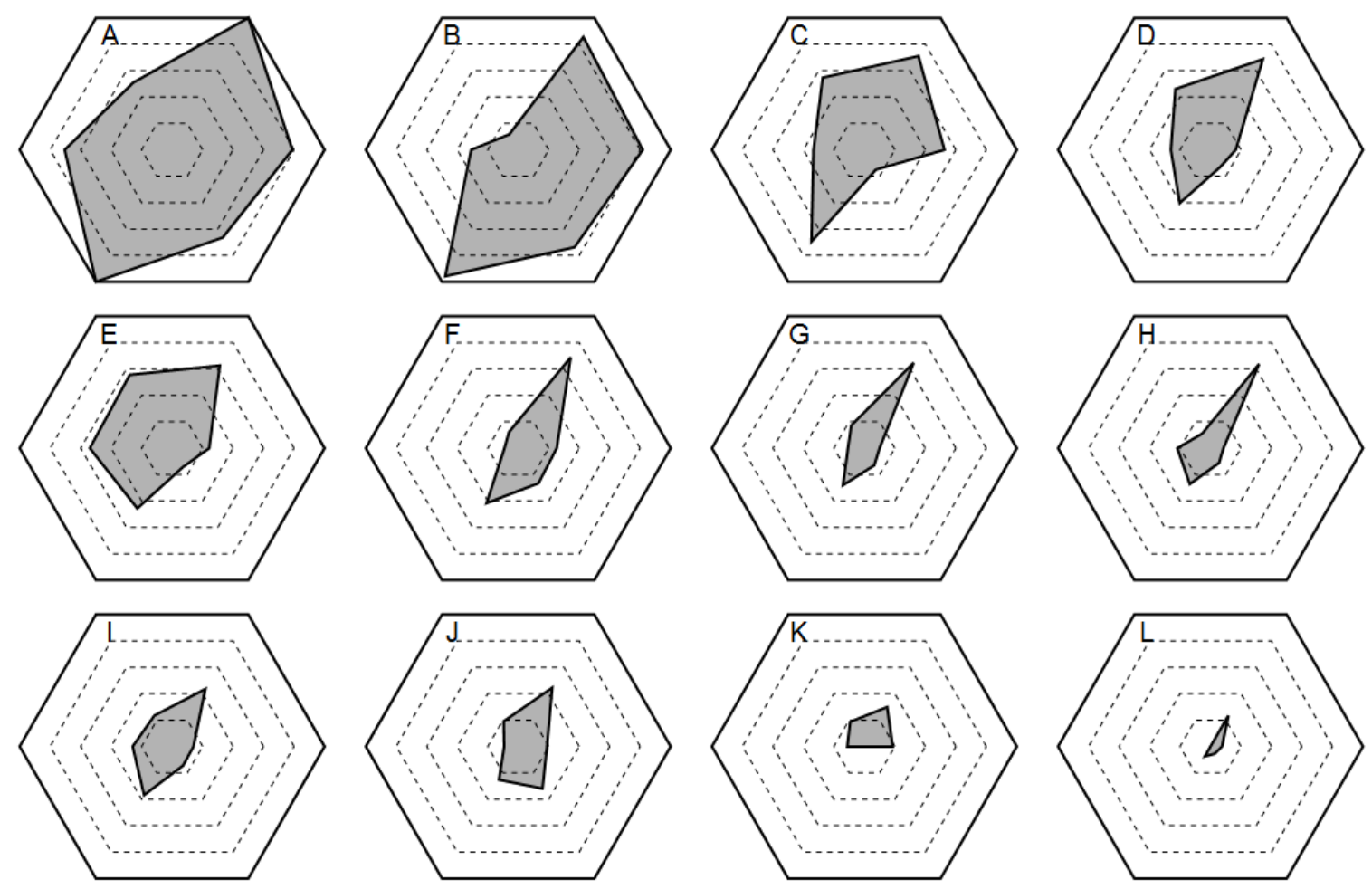

Figure 4. The six-dimensional vector $\mathrm{G}=(\mathrm{EF}, \mathrm{Ew}, \mathrm{Es}, \mathrm{BF}, \mathrm{Bw}, \mathrm{Bs})$ consisting of facets $\mathrm{F}, \mathrm{W}$ and $\mathrm{S}$ of $\mathrm{E}$ - and $\mathrm{B}$ fields represented as hexagonal spider webchart. Coding as in Figure 3, where cases A, C and $\mathrm{H}$ are shown as examples.

As is seen in Figure 4 there is lot of variation in the centralities and in the richness of content. The networks $\mathrm{A}, \mathrm{B}$ and $\mathrm{C}$ are the ones with richest content and network $\mathrm{A}$ in particular is quite wellbalanced having all facets strong. However, quite many networks have very low richness of content, in particular $\mathrm{K}$ and L. It should be noted that although these networks have also lower number of links in comparison to e.g. A-C, the very low richness results mainly form poor epistemic justification of the links in the concept network; the knowledge represented in these networks is meagre. Some of the networks, such asF, G and $\mathrm{H}$ have strong EF facet but very other facets remain quite weak. These are clear examples of force-dominant conceptions of E-field. It is noteworthy that only in the richest cases A and B also all B-field facets are strong, in C, which is the third in richness, only F and S facets remain strong. In all other cases the B-field facets remain weak.

Additional information of the facets is provided by the relative centralities, which are case-by-case normalized centralities instead to centralities normalized to sample maximum. The relative centralities do not depend on the richness of the content are well suited to reveal the systematic dependencies on how phenomenological support is distributed and what percentage the given facet constitutes the field concept in different cases A-L. These relative centralities are shown in Figure 5 for all 12 cases. 

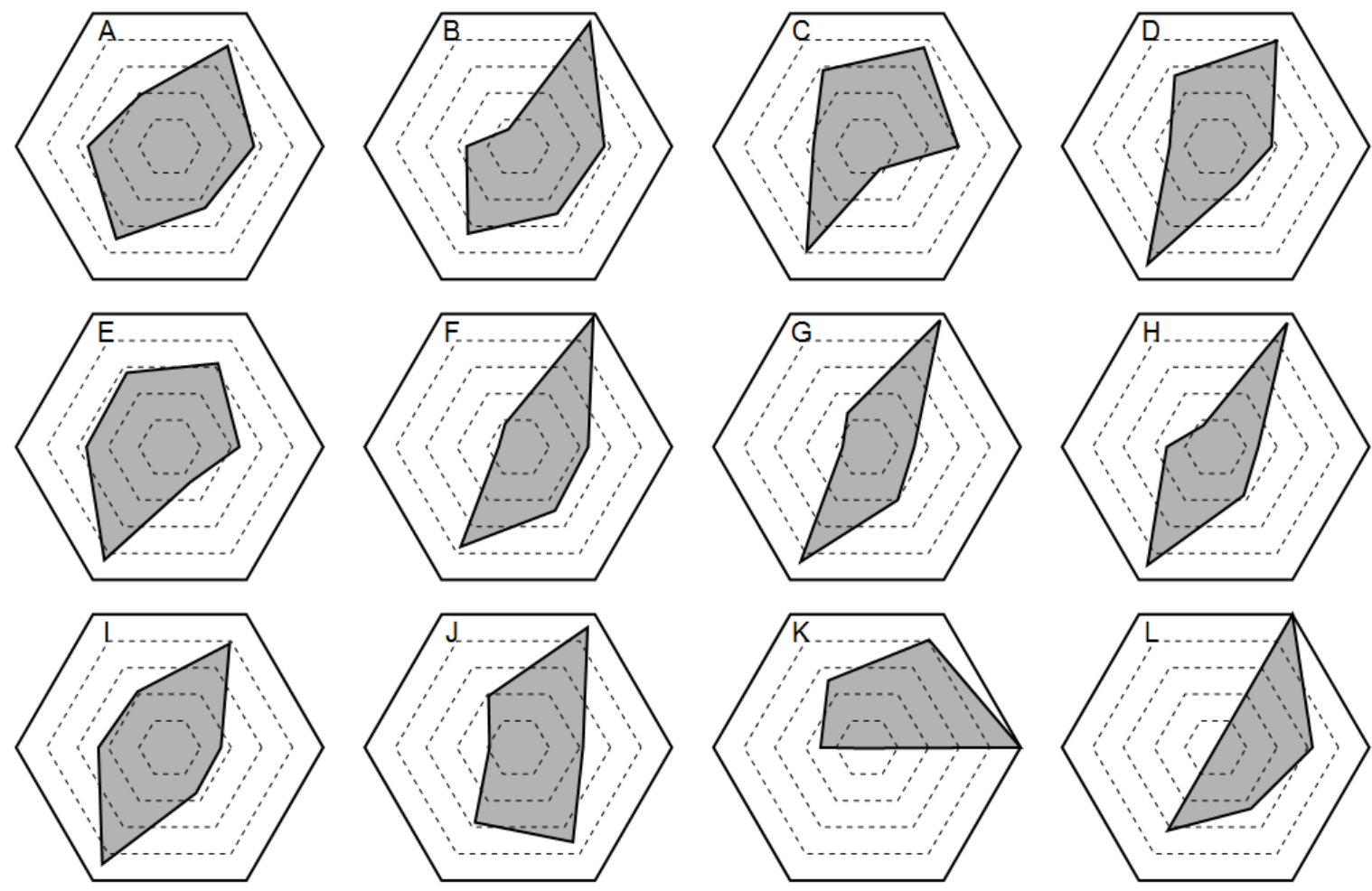

Figure 5. Normalized facets of E-field and B-field. Each case A-L is normalized to total centrality 1 for all six centralities. Otherwise as in Figure 4.

As can be seen, the normalized centralities display more regular behaviour in comparison to nonnormalized centrality. The regularity found in relative values of centralities suggests that although students may have very different amount of content knowledge, the ways to understand the support and facets of field concepts have more regularity, not related to the content richness. From the relative centralities it is clear that nearly all some students conceive E and B strongly force based, students that produced the networks F-I and L, solely based on force-facet F.

The results in Figures 4 and 5 indicate that the representations of declarative knowledge of field concepts with the highest richness of content (cases A-C with large values of total centrality) have also balanced facets, the cases with intermediate richness (D and F-I) tend to reflect forcebased understanding and give relatively less weight on energy and source based understanding. This probably reflects the fact that the forcebased introduction of field concept is the standard starting point of instruction in introductory university level. In the cases J-K where the content richness is lowest the relative distribution of weights appears to be varied. One simple interpretation is that with increasing richness of content also the ability to express different facets increases, while in case of intermediate richness only force-facet is successfully adopted. The cases with the lowest richness rely on very arbitrary connections and appear thus quite random in respect how different facets are weighted.

Finally, it is of interest to contrast the result of facets to the result obtained by standardized conceptual test. The concept networks which have been used here probably require that basic content knowledge is mastered in level which is often called "conceptual understanding" but still differs much from what is measured by so called conceptual test. The conceptual test are typically qualitative, quiz-type, multiple choice problems, with one correct answer and two or more distractors. The conceptual understanding of facets is then apparently very different (requiring the epistemic understanding and construction of coherent arguments) to conceptual understanding the tests are measuring. Nevertheless, the conceptual tests can be taken as indicators a kind of threshold 
for higher levels of understanding. Here, to check this assumption we also carried out Conceptual Survey of Electricity and Magnetism, the CSEM-test which is measures the conceptual understanding of basic concepts in electricity and magnetism through 32 questions (Maloney et al., 2001). Without going into details, we accept here the view that CSEM operationalizes a certain kind of conceptual understanding. Typically, university students of calculus based courses have $31 \%$ and students in algebra based courses $25 \%$ correct answers in the pre-test results, while they have in post-test $47 \%$ and $44 \%$ correct answers, respectively (Maloney et al., 2001).

In the CSEM test, however, only a certain fraction is connected to topics discussed here. These topics are comprised by CSEM items 1-6, 8-13, 15, 19 and 20 (item group gE in what follows) for electric fields and items 21-28 and 31 (item group gв) for magnetic fields (for details see Maloney et al. 2001). The items were selected on basis that they correspond most directly the topics in concept networks. Some otherwise suitable items were excluded because we estimated them to be strongly biased to rote learning of certain conventions (e.g.rules to determine direction of magnetic force) and thus of little relevance in present context.

The CSEM was carried out as pre- and post-tests. The pre-test results (scores normalized to maximum of correct answers) for gE, gвand how they correlate with norm (content richness, see Table 2) $\Gamma_{\mathrm{E}}$ and $\Gamma_{\text {в }}$ for E-field facets and B-field facets, respectively are shown in Figure 6 (left panel), as well the total correlations between $\mathrm{g}_{\mathrm{E}+\mathrm{B}}$ and for total richness $\Gamma_{\mathrm{E}+\mathrm{B}}$ of all $\mathrm{E}$ and $\mathrm{B}$ facets taken together. All three datasets revealing the correlations are shown in Figure 6 (left panel). The correlations between richness and pre-test CSEM scores are weak in all cases;at best 0.40 with p-value 0.19 (not significant) with Spearman-rho test between datasets $g_{\mathrm{E}+\mathrm{B}}$ and $\Gamma_{\mathrm{E}+\mathrm{B} a n d}$ CSEM pre-test scores. This indicates that the pre-test CSEM score and therefore, the conceptual understanding as it is exemplified by qualitative problems, is not good predictor for success in expressing rich declarative content knowledge which is epistemically well justified.

The picture changes when instead of scores the gains in CSEM are measured. The gain G is defined as ratio of change from post- to pre-test scores to maximal available change

$$
G=\frac{\text { (post-test score })-(\text { pre-test score })}{\text { (maximum score) }-(\text { pre-test score })}
$$

The gain $\mathrm{G}$ is thus a measure of learning between the pre- and post-test. All three datasets correlating the gains $G_{E}$ for item group ge and and $G_{B}$ for gв with $\Gamma_{\mathrm{E}}$ and $\Gamma_{\mathrm{B}}$ as well as $\mathrm{G}_{\mathrm{E}+\mathrm{B}}$ for g $\mathrm{g}_{\mathrm{E}+\mathrm{B}} \mathrm{With}_{\mathrm{E}} \Gamma_{\mathrm{E}+\mathrm{B}}$ are shown in Figure 6 (right panel). The correlation of the gains $G_{\mathrm{E}}$ and Gвwith $\Gamma_{\mathrm{E}}$ and $\Gamma_{в}$ ваre now 0.44 $(p=0.16)$ and $0.35(p=0.11)$, respectively. These correlations are not significant, either. However, when gains $\mathrm{GE}_{\mathrm{E}+\mathrm{B} t a k i n g}$ into account all items $\mathrm{g}_{\mathrm{E}+\mathrm{B}}$ are correlated with total richness $\Gamma_{\mathrm{E}+\mathrm{B}}$ of all $\mathrm{E}$ and $\mathrm{B}$ facets taken together the Spearman-rho becomes $0.48(\mathrm{p}=0.11)$. When one outlier is ignored, the correlation improves to $0.67(\mathrm{p}=0.02)$, which is a significant correlation. And additional test with Kendall-tau rank correlations gives similar indications, and only between $\mathrm{g}_{\mathrm{E}+\mathrm{B}}$ and $\Gamma_{\mathrm{E}+\mathrm{B}}$ Kendall-tau is significant, with $0.52(\mathrm{p}=0.03)$.

Although the sample is small, the results in Figure 6 suggest that good CSEM test results are required to produce rich content of field-facets, but on the other hand, good CSEM results do not guarantee that a student can produce a good relational representation (a rich network). This suggest that CSEM and concept nets represent different dimensions of mastery of content knowledge, where CSEM gauges pre-requirements to produce a good network, but network as such, sets higher metacognitive demands not accessed by CSEM. In addition, Figure 6 suggests that producing the network is also useful for learning. 

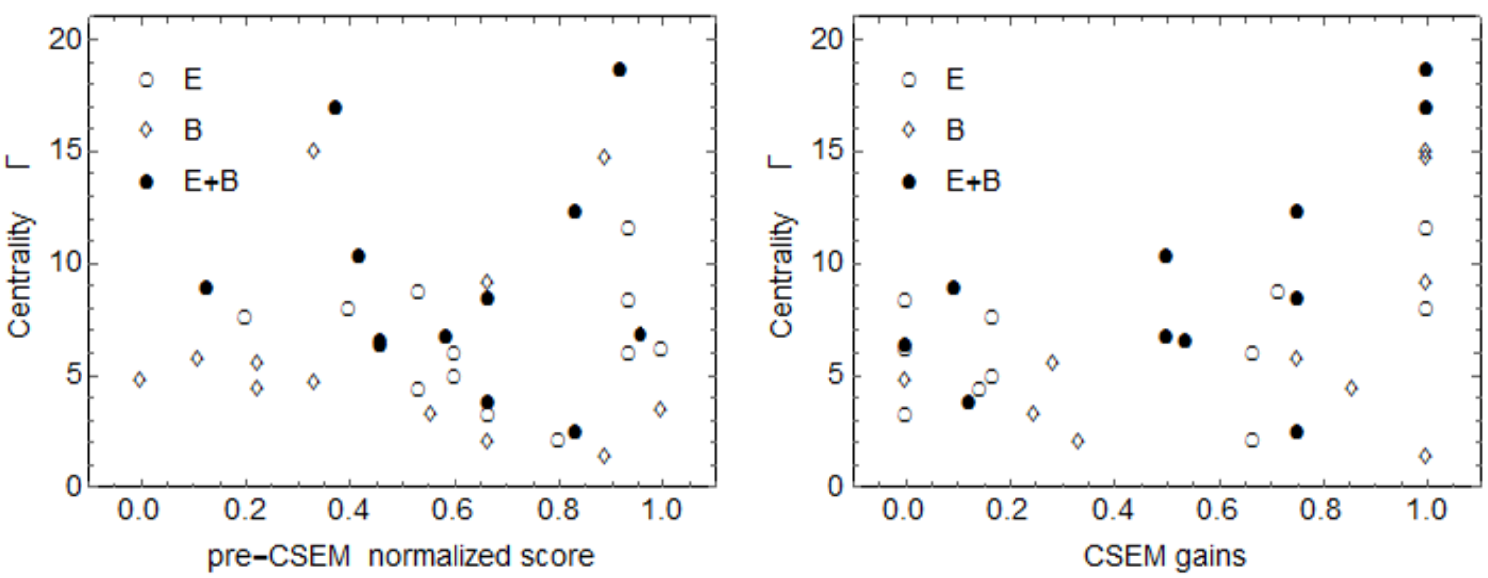

Figure 6. Correlations between centrality $\Gamma$ calculated for of $E$ and $B$ separately and for $E$ and $B$ together (see legends in figure) with CSEM pre-test score (left panel) and gains (right panel).

\section{Discussion and Conclusions}

Several studies have explored how students learn different aspects and properties of electric field concept, some studies have also addressed the learning of magnetic field (Furió\& Guisasola, 1998; Guisasola et al., 2004; Galili, 1995; Viennot \& Rainson, 1992; Törnqvist et al., 1993; Pocovi \& Finley, 2002; Albe et al., 2001). However, all the existing studies have focused on some isolated aspect of field concepts. Here we have approached the question how students learn the field concepts from a viewpoint of declarative (expressed and communicated) knowledge and its epistemic justification. We have used concept networks as representational tools that student can use to express their ideas of the relationships between concepts and other conceptual elements. The concept networks are constructed so that epistemic justification of knowledge represented in the networks is taken into account (Nousiainen, 2013). The approach pays attention on the relationships between concepts and their conceptual support. Towards that we have developed network theoretical methods, which make the complex, global analysis possible (Koponen \& Nousiainen, 2014). Our approach allows us to develop an integrated perspective which sees concepts as many faceted and many dimensional structures.

In analysis of many dimensionality of the electric (E) and magnetic (B) field concepts we have focused on force, energy and source (electric charge in case of E-field and electric current in case of B-field), which can be taken as the facets (or dimensions) that support the formation of the field concepts. Force, energy and source are also the phenomenological founding that can be discerned in textbook introduction on the field concepts and on the historical development of the field concept. The analysis how E- and B-field concepts are supported by the force, energy and source dimensions is based on the analysis of linked relationships of field concept to other concept that are involved in construction of the meaning of field concepts, mainly through experiment and models, that students introduce in their concept networks.

The result shows that there are significant differences between students' ability to make connections between different phenomenological basis of field concepts, as well as in the richness of content knowledge the students display in their concept networks. The richness of content in concept networks may differ by a factor of four, when global connections are taken into account. The most notable finding is that with increasing richness of knowledge the relative proportions of E-field facets become quite balanced, so that students who command rich content also display balanced understanding of force-, energy and source based facets of E-field. Students, who have less content knowledge, tend to favour forcebased conceptions. In case of B-field, situation is different. In all cases 
magnetic field show strong bias to force (or torque) based support. Only in the cases of richest content the sourcebased support is also strong when force-facet is strong. This probably reflects the centrality of Ampère's law in many study materials and textbooks (compare Majidi, 2014).

The results indicate that the most evident features for both electric and magnetic field provided in the textbooks are strongly biased by force which is in many study material and textbooks used as the basis of introducing these concepts. It seems that only when students gain richer background knowledge, they learn more ways to make connections between concepts, more experiments and models which support the concepts, and only then the other facets of the field concepts become enriched and more balanced way emerges. The centrality of traditional forcebased introduction of field concepts have been criticized and alternatives are proposed to use microscopic models (i.e. essentially sourcebased) introductions to field concepts (Chabay \& Sherwood, 2006). Also in light of the results presented here these suggestions are indeed well-motivated and re-structuring of traditional course contents with strong forcebased views need reconsideration.

The connection of the richness of declarative knowledge in relation to conceptual understanding as it is reflected in gains in conceptual survey of electricity and magnetism (CSEM, see Maloney et al., 2001) test deserves also attention. The correlation of pre-test scores with richness of content in networks is not clear, although the $25-30 \%$ threshold of correct answer seems to be a prerequisite to adequate constriction of epistemically well-justified concept network (reflected as high enough centralities of facets). However, good CSEM results in pre-test do not guarantee success in concept network construction; a student's CSEM result can be high but concept network is meagre and weak. When gains in CSEM results are correlated with richness of the content of fieldconcepts (centrality of E- and B-field taken together) the correlation is found to significant. This can be interpreted so that the construction of network has facilitated further learning and lead to increased conceptual understanding as it is measured by CSEMtest. In one case contained in the study the gains are astonishingly high. However, also in case of gains the conceptual understanding as measured by CSEM appears to be the prerequisite but not sufficient alone to reach rich content and well-balanced fieldconcepts as monitored by the concept networks.If the interpretation is correct, it suggests that if higher meta-level abilities and rich understanding of physics concepts (conceptual understanding) is the goal, the conceptual tests such as CSEM are too limited to assess whether or not the goals in knowledge organization and its epistemic justification are reached. At best, the conceptual test tell the necessary requirement but provide not yet the sufficient conditions. This, of course, is often made remark by those who utilize the tests. It should be noted that although the interpretation of the correlation of gains and CSEM-test are suggestive, the results are not conclusive and more convincing conclusion would require larger sample.

The goals of knowledge organization, its epistemic justification and coherent argumentation, required to construct a rich concept network, are of course central for physics teacher education and core competencies of a teacher. For these central competencies we have in this study provided some new approaches to augment the repertoire of assessment tools to meta-level which can in significant way support and extendthe assessments based of conceptual surveys. In research methodological level the present work demonstrates that in order to reveal the important and relevant features in learning complex, many faceted concepts attention must be paid on developing sophisticated enough methods of research. In practical level, the results show that although it is possible for student to develop a balanced and rich understanding how different aspect or facets of the field concepts are constructed, this does not happen to be case for all students. It may be that most current textbooks do not actually support explicitly formation of such picture, although they implicitly contain all material needed for such development. Therefore, paying attention explicitly to different facets of field concepts may beneficial forboth teaching and learning. 


\section{References}

Albe, V., Venturini, P., and Lascours, J., (2001). Electromagnetic Concepts in Mathematical Representation of Physics. Journal of Science Education and Technology, 10(2), 197-203.

Chabay, R., and Sherwood, B., (2002).Matter \& Interactions: Electric and Magnetic Interactions.USA: John Wiley \& Sons, Inc.

Chabay, R., and Sherwood, B., (2006). Restructuring the introductory electricity and magnetism course. American Journal of Physics, 74(4), 329-336.

Chang, W., (2011). Integrating electrostatics with demonstrations and interactive teaching.American Journal of Physics, 79(2), 226238.

Darrigol, O., (2000). Electrodynamic from Ampère to Einstein. New York, USA: Oxford University Press.

Estrada, E.,(2012).The Structure of Complex Networks. Oxford, UK: Oxford University Press.

Feynman, R., Leighton, R., and Sands, M.,(1964). The Feynman Lectures on Physics, vol. II. Massachusetts, USA: AddisonWeasley.

Furió, C., and Guisasola, J., (1998). Difficulties in learning the concept of electric field. Science Education, 82(4), 511-526.

Galili, I.,(1995). Mechanics Background for Students' Misconceptions in Electro-Magnetism. International Journal of Science Education,17(3), 371-387.

Guisasola, J., Almudi, J., Salinas, J., Zuza, K., and Ceberio, M., (2008). The Gauss and Ampere laws: different laws but similar difficulties for students learning. European Journal of Physics, 29(5), 1005-1016.

Guisasola, J., Almudi, J.M., and Zubimendi, J.L., (2004). Difficulties in learning the introductory magnetic field theory in the first years of university. Science Education, 88(3), 443-464.

Halliday, D., Resnick, R.,and Walker, J., (2005).Fundamentals of Physics. USA: John Wiley \& Sons, Inc.

Hoyningen-Huene, P., (1993).Reconstructing Scientific Revolutions: Thomas S. Kuhn's Philosophy of Science. Chicago, USA: Chicago University Press.

Kelly, J., and Takao, A., (2002). Epistemic levels in argument: An analysis of university oceanography students' use of evidence in writing. Science Education, 86(3), 314-342.

Knight, R.D.,(2008). Physics for Scientists and Engineers: A Strategic Approach. San Francisco, USA: Pearson Addison-Wesley. Koponen, I., and Nousiainen, M., (2013). Pre-service Physics Teachers' Understanding of the Relational Structure of Physics Concepts: Organising Subject Content for Purposes of Teaching. International Journal of Science and Mathematics Education, 11(2), 325-357.

Koponen, I., and Nousiainen, M., (2014). Concept Networks in Learning: Finding Key Concepts in Learners' Representations of the Interlinked Structure of Scientific knowledge. Journal of Complex Networks, 2(2), 187-202.

Krathwohl, D., (2002). A revision of Bloom's taxonomy: an overview. Theory into practice, 41(4), 212-218.

Kuhn T. S., (1996/1962). The Structure of Scientific Revolutions (3rd ed.). Chicago, USA: University of Chicago Press.

Majidi, S., (2014). A Comparison between the Knowledge Organization of University Physics Teachers and the Textbooks They Use for Their Teaching Purposes: Biot-Savart Law and Ampere's Law.International Journal of Science and Mathematics Education, 12(6), 1281-1314.

Majidi, S.,and Mäntylä, T.,(2011).Knowledge Organization in Physics Textbooks: A Case Study of Magnetostatics. Journal of Baltic Science Education, 10(4), 285-299

Maloney, D., O'Kuma, T., Hieggelke, C.,and Van Heuvelen, A., (2001). Surveying students' conceptual knowledge of electricity and magnetism. American Journal of Physics, 69(7), 12-23.

Nousiainen, M., (2013). Coherence of Pre-service Physics Teachers' Views of the Relatedness of Physics Concepts. Science \& Education, 22(3), 505-525.

Pocovi, M.,and Finley, F., (2002). Lines of Force: Faraday's and Students' Views. Science \& Education, 11(5), 459-474.

Sampson, V., and Clark, D., (2008). Assessment of the Ways Students Generate Arguments in Science Education: Current Perspectives and Recommendations of Future Directions. Science Education, 92(3), 447-472.

Sandoval, W., and Millwood, K., (2005). The Quality of Students' Use of Evidence in Written Scientific Explanations. Cognition and Instruction, 23(1), 23-55.

Savelsbergh, E., de Jong, T., and Ferguson-Hessler, M., (2011). Choosing the right solution approach: The crucial role of situational knowledge in electricity and magnetism. Physical Review Special Topics - Physics Education Research, 7(010103), 1-12.

Smith,C., and Wise,M. N., (1989).Energy and Empire. A biographical study of Lord Kelvin. Cambridge, UK: Cambridge University Press.

Törnkvist, S., Pettersson, K.-A., and Tranströmer, G., (1993). Confusion by representation: On student's comprehension of the electric field concept. American Journal of Physics, 61(4), 335-338.

Viennot L., and Rainson S., (1992). Students' reasoning about the superposition of electric fields. International Journal of Science Education, 14(4), 475-487.

Whittaker, E., (1910). A History of the theories of aether and electricity. Dublin, Ireland: Longman, Green and Co.

Yin, Y., Vanides, J., Ruiz-Primo, M. A., Ayala, C. C., and Shavelson, R. J., (2005). Comparison of two concept-mapping techniques: Implications for scoring, interpretation, and use. Journal of Research in Science Teaching, 2(2), 166-184.

Young, H., and Freedman, R.,(2004). Sears and Zemansky's university physics. San Francisco, USA: Pearson Addison-Weasley. 